\title{
Screening for Glucose-6-Phosphate Dehydrogenase deficiency using quantitative analysis of G6PD activity: A pilot study to expand the newborn screening panel in Sri Lanka
}

\author{
Hettiarachchi $\mathbf{M}^{1}$, Amarasena $\mathrm{S}^{2}$ \\ ${ }^{I}$ Nuclear Medicine Unit, Faculty of Medicine, University of Ruhuna, Galle, Sri Lanka. \\ ${ }^{2}$ Department of Paediatrics, Faculty of Medicine, University of Ruhuna, Galle, Sri Lanka. \\ Correspondence: Prof. Manjula Hettiarachchi \\ email:manjulah@ruh.ac.lk \\ (D) https://orcid.org/0000-0003-1203-1368
}

\begin{abstract}
Introduction: Glucose-6-phosphate dehydrogenase (G6PD) deficiency affects over 400 million individuals worldwide and commonly found in Asia and in the Mediterranean region. This study was aimed to assess the feasibility of incorporating G6PD screening to the existing newborn screening panel in Sri Lanka.
\end{abstract}

Methods: Dried blood spot samples ( $\mathrm{n}=8795)$ received during a period of two months consecutively were subjected to the initial analysis and the G6PD activity was calculated by a quantitative analysis of comparing the sample to the control with a known activity using the haemoglobin normalization method. All babies with G6PD residual activity $<2.5 \mathrm{U} / \mathrm{gHb}$ were recalled $(\mathrm{n}=215)$ for a second sample after 6 months and analyzed using the same assay protocol in order to validate the assay

Results: Analysis of second samples revealed that 58 babies ( 36 males and 22 females) continued to have $<2.5$ $\mathrm{Ug} \mathrm{Hb}$ activity. The incidence of G6PD deficiency in this selected population was 1 in 155 . Range of the measured G6PD activity was $0.5-13.8 \mathrm{U} / \mathrm{g} \mathrm{Hb}$. Inter assay variation was $2.7 \%(13.81 \mathrm{U} / \mathrm{gHb})$. Hambantota and Matara districts of the Southern province, which were previously known as malaria endemic areas, reported a higher prevalence.

Conclusions: The incidence of G6PD deficiency is reported for the first time among neonates in Sri Lanka. However, the cut-offs we have defined at 6 months may be at a disadvantage with high attrition, but it saves the cost of additional genetic analysis.

Key words: Newborn screening, glucose-6-phosphate dehydrogenase (G6PD), enzyme activity, dried blood spot, haemoglobin normalization

\section{Introduction}

Newborn screening (NBS) is the largest genetic testing effort and is considered as one of the ten great public health achievements during the first 10 years of the $21^{\text {st }}$ century (1). Tests used in NBS are designed to detect asymptomatic newborns at risk of a disease from those who are not at risk. Effective screening of newborns, combined with follow-up confirmatory diagnostic testing and treatment, helps prevent morbidity and mortality (2).
Glucose-6-Phosphate-Dehydrogenase (G6PD) is an important enzyme in the hexose monophosphate oxidative pathway where it plays an essential role in reducing nicotinamide adenine dinucleotide phosphate $(\mathrm{NADP}+)$ to $\mathrm{NADPH}+$. NADPH+ protects red cells from oxidative damage. In a reaction catalyzed by G6PD, electrons generated by the conversion of glucose-6-phosphate to 6phosphogluconate are transferred to NADP+ (3). G6PD deficiency can lead to an increased risk and 
earlier onset of neonatal hyperbilirubinaemia or may present later in life with acute haemolytic crises. Intermittent exacerbations of acute intravascular haemolysis occur after exposure to certain drugs like antimalarial or sulfonamides, food stuffs like fava beans, or with infections such as viral respiratory infections, hepatitis, and bacterial pneumonia (4). In certain populations, hyperbilirubinaemia secondary to G6PD deficiency results in an increased risk of kernicterus and death, whereas in others, this has not been observed (5). This may reflect mutations that are specific to ethnicity, altering the susceptibility to develop kernicterus. The explanation for the higher frequency of clinical manifestations of G6PD deficiency in the neonatal period is due the limited capacity of the neonatal liver to metabolize and eliminate bilirubin, and the limited capacity of the red blood cells of the neonate to withstand oxidative stress (6).

Neonatal screening for G6PD deficiency utilizing the semiquantitative fluorescent spot has been carried out in many countries, including Malaysia (7) and India (4). Its incidence is different in different ethnic groups. Though the exact incidence is not known, various studies have reported an incidence ranging 2-27\% in different communities in India (8).

The quantitative measurement of G6PD coupled to a simultaneous evaluation of the haemoglobin $(\mathrm{Hb})$ content in the sample, expressing the results in Units/gram hemoglobin $(\mathrm{U} / \mathrm{g} \mathrm{Hb})$. Hb normalization will allow for correction of all samples against a selected standard or control used in the test by 'normalizing' all analyte values so that they correspond to the same quantity of sample (7) had established the normal reference range for G6PD enzyme activity level with more sensitive in detecting individuals with partial G6PD levels.

The newborn screening program of Sri Lanka is well established but confined to screening for congenital hypothyroidism (CH) (9). The aim of the study was to assess the feasibility of incorporating G6PD screening to the screening panel.

\section{Methods}

The ethical approval for the study was obtained from the Ethics Review Committee of Faculty of Medicine, University of Ruhuna. The study was conducted in 4 out of 15 districts where the NBS program has been functioning for over 5 years. Since samples are sent from these districts for confirmation of $\mathrm{CH}$, an already established mechanism exists to receive samples for screening of G6PD.

Dried blood spot samples received during two consecutive calendar months (May to June 2017) from these 4 districts were subjected to the initial analysis of G6PD enzyme levels.

The G6PD screening assay kit manufactured by MP Biomedicals Germany GmbH (Ref. 07G580307) which was utilized in the analysis, has G6PD, which in the presence of NADP + , catalyses the oxidation of glucose-6-phosphate to 6-phosphogluconate. The NADPH + reacts with a color reagent in which a tetrazolium salt gets reduced to produce a distinct color. This color is measured colorimetrically at the optical density (OD) of $550 \mathrm{~nm}(500-570 \mathrm{~nm})$ and is directly proportional to the concentration of G6PD present in the sample. The reduced tetrazolium salt can be measured in endpoint mode using two measurements (at zero minute and 10-15 minutes later) while the microplate remains in the incubator. The G6PD activity of the blood sample was calculated by comparing the rates of the sample to rate of the normal control with known G6PD activity and measuring the haemoglobin level of the sample ( $\mathrm{Hb}$ Normalization) at the OD of $405 \mathrm{~nm}$. The following formula (Greek Patent \# 1003227; International Patent Pending) expresses each subject's G6PD levels in U/g of Hb.

$\left(\delta\right.$ OD sample $\left.550 \mathrm{um}^{1}\right) /\left(\delta\right.$ OD control $\left.550 \mathrm{~nm}^{2}\right)$

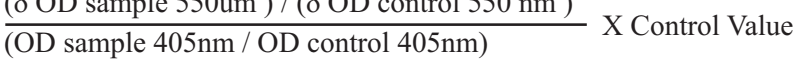

${ }^{1} \delta$ OD sample $550 \mathrm{~nm}=$ Final OD sample $550 \mathrm{~nm}$ at $15 \mathrm{~min}$ - Initial OD sample $550 \mathrm{~nm}$ at $0 \mathrm{~min}$ ${ }^{2} \delta$ OD control $550 \mathrm{~nm}=$ Final OD control $550 \mathrm{~nm}$ at $15 \mathrm{~min}-$ Initial OD control $550 \mathrm{~nm}$ at $0 \mathrm{~min}$

In the above formula endpoint mode was used for the measurement of G6PD activity and analyzed in singleton. The difference between the final and the initial OD reading was used. Control value was provided by the manufacturer.

G6PD activity levels were reported as totally deficient, partially deficient or normal in order to reassess in a second sample after 6 months. Babies with residual activity range $<2.5 \mathrm{U} / \mathrm{g} \mathrm{Hb}$ during initial screening were informed and recalled a second dried blood spot sample after 6 months to validate the G6PD enzyme activity. All these 
infants' data were traced from newborn screening information system database (www.nsisd.ruh.ac.lk) and parents were contacted through mobile phone or by a letter to parents to collect the second sample after 6 months (i.e., December 2017 to February 2018). Parents were given instructions to meet the consultant pediatrician of the respective hospital (where the baby was delivered) and to obtain a fresh heel prick blood sample to be delivered to the laboratory through the hospital sample delivery system.

Data were compared at district levels to give incidence rates.

\section{Results}

Altogether, there were 8795 dried blood spot samples (4680 in May and 4115 in June) collected during the study period from hospitals in the selected districts (Table 1). The range of the measured G6PD activities was $0.5-13.8 \mathrm{U} / \mathrm{gHb}$ with inter assay variation of $2.7 \%(13.81 \mathrm{U} / \mathrm{gHb})$. When the limit of sensitivity to represent a change in absorbance is $1 \mathrm{mOD} / \mathrm{min}(0.001 / \mathrm{min})$ of G6PD activity at $550 \mathrm{~nm}$, the detectable level of G6PD activity will increase by $0.9 \mathrm{U} / \mathrm{g} \mathrm{Hb}$. Using kinetic mode and four decimal displays, the detection limit becomes $0.3 \mathrm{U} / \mathrm{gHb}$. Subjects were classified according to the obtained result as follows: Totally deficient: residual activity range $<2.5 \mathrm{U} / \mathrm{gHb}$; partially deficient : residual activity range $2.6-6.5 \mathrm{U} / \mathrm{gHb}$; normal: with residual activity $>6.6 \mathrm{U} / \mathrm{gHb}$. There were 215 samples with residual activity $<2.5 \mathrm{U} / \mathrm{gHb}$ on initial analysis.
For the second analysis we received only 114 samples resulting nearly $50 \%$ non-respondent rate (Table 1).

Analysis of second samples revealed that 58 babies (36 males and 22 females) continued to have low residual activity $(<2.5 \mathrm{Ug} / \mathrm{Hb})$ after 6 months of life. The incidence of G6PD deficiency during the study period in this selected population was 1 in 155 . Highest percentage (45\%) of positive babies was from Hambantota District and the lowest percentage (2\%) was from Ratnapura District.

\section{Discussion}

We report the incidence of G6PD deficiency for the first time among Sri Lankan neonates. In the context of G6PD screening, the most common approach has been to include G6PD screening within other screening programs, typically $\mathrm{CH}$ screening (10). The gene, which encodes for G6PD is located on the X-chromosome (7). Since boys inherit the $\mathrm{X}$-chromosome only from their mothers, it is known that mothers are also partially deficient in G6PD. Further, it revealed that intermediate enzymatic activity is present in Greek and Albanian mothers and in the cord blood of their female newborns (11). For many years, G6PD deficient heterozygotes were not regarded as being at risk (12). However, Kaplan et. al, in 2001 (13) have shown that heterozygotes with minimal haemolysis are also at similar risk for hyperbilirubinaemia as G6PD homozygotes in the newborn period.

Table 1: G6PD Screening results

\begin{tabular}{|c|c|c|c|c|c|c|}
\hline \multirow{2}{*}{ District } & \multicolumn{2}{|c|}{ No. of samples } & \multirow{2}{*}{$\begin{array}{l}\text { Screening } \\
\text { Positive }^{1} \\
\end{array}$} & \multicolumn{2}{|c|}{ Repeat analysis ${ }^{2}$} & \multirow[t]{2}{*}{ Incidence } \\
\hline & May & June & & Responded & Confirmed $^{3}$ & \\
\hline Ratnapura & 1431 & 1210 & 9 & 3 & 1 & 1 in 2641 \\
\hline Matara & 917 & 690 & 53 & 27 & 18 & 1 in 89 \\
\hline Galle & 1499 & 1566 & 61 & 30 & 13 & 1 in 236 \\
\hline Hambantota & 914 & 740 & 92 & 54 & 26 & 1 in 64 \\
\hline Total & \multicolumn{2}{|c|}{8967} & 215 & 114 & 58 & 1 in 155 \\
\hline
\end{tabular}

${ }^{1}$ G6PD activity with $\mathrm{Hb}$ normalization $<2.5 \mathrm{U} / \mathrm{gHb}$ on first dried blood sample

${ }^{2}$ Responded to repeat analysis after 6 months

${ }^{3} \mathrm{G} 6 \mathrm{PD}$ activity with $\mathrm{Hb}$ normalization $<2.5 \mathrm{U} / \mathrm{gHb}$ on second dried blood sample

${ }^{4}$ Number of confirmed G6PD deficient babies per number of babies delivered during the study period 
The diagnosis of G6PD deficiency is made by a quantitative spectrophotometric analysis or, more commonly, by a rapid fluorescent spot test detecting the generation of NADPH from NADP+ $(6,14)$. Hemoglobin normalization is a procedure which could be used in a variety of platforms and tests. We believe that the implementation of this procedure would greatly benefit newborn screening. In this aspect, haemoglobin normalization will allow for correction of all samples against a selected standard or control used in any assay by "normalizing" all analyte values so that they correspond to the same quantity of sample (15). It has been challenging to define a single universal normal (100\%) G6PD activity value, so that classification of the G6PD status of an individual is defined as the percentage of a normal value determined locally (10). G6PD phenotype classifications for purposes of test performance evaluation were described in 2016 by the WHO (16) where males with less than $30 \%$ activity are considered as deficient and females with less than $30 \%, 30-80 \%$, and greater than $80 \%$ G6PD activity are considered G6PD deficient, intermediate, and normal, respectively. Further, it was mentioned that the cost-effectiveness of screening, the frequency and severity of G6PD deficiency in a specific population, availability and efficacy of appropriate diagnostic options, and the capacity of the health system to provide appropriate counseling to parents and providers (17) should be considered prior to implementing such robust screening program.

In this study, 215 neonates were detected with deficient G6PD levels out of approximately 9,000 neonate samples on first screening. Of them, 101 neonates could not be followed up at 6 months for repeat sampling. This high attrition rate could be due to several reasons. There was no pretest counseling as G6PD tests were done in samples received for $\mathrm{CH}$ screening using the second blood spot. There were no dropouts in $\mathrm{CH}$ screening, but the recall takes place immediately within few days of the receipt of the samples and there is adequate pretest education and counseling in the $\mathrm{CH}$ screening program. This may change if the pretest counseling process is introduced for G6PD deficiency program in order to have a high recall rate (9). This was an initial attempt to identify the presence of G6PD deficiency in a population study and study the ability to test for G6PD deficiency and feasibility of introducing the G6PD screening to the current NBS program. Further, we did not educate and seek the assistance from public health midwives to trace these babies. This would be a classic example for any screening program being unable to achieve its objectives as a result of not being incorporated to the existing public/national health systems. However, G6PD deficiency was diagnosed in 58 (27\%) neonates at confirmatory testing showing that, it is feasible to introduce G6PD deficiency screening into the NBS program in the country as the existing laboratory has the capability to handle it.

There were studies that attempted to determine the prevalence of G6PD deficinecy in regional level in Sri Lanka (18) among adult hospital attendees. However, all these studies reported that more females were affected than males. In our study, out of the 58 deficient neonates, 36 were males while 22 were females with the ratio of $1.6: 1$. In a two year follow up study in India, male to female ratio of G6PD deficient neonates was $5.4: 1$ (4). In two studies conducted in India male to female ratio of G6PD deficient neonates were $1: 1$ (19) and 3: 1 (20) respectively, even though G6PD deficiency is expected to be rare in females as it is an X-linked recessive disorder.

G6PD deficiency is currently considered in the differential diagnosis of neonates who develop hyperbilirubinaemia within the first 24 hour of life, those who have a sibling with history of jaundice and who have bilirubin levels greater than the $95^{\text {th }}$ percentile (4). Clinicians investigate these high-risk children for G6PD deficiency after a few months. However, G6PD deficiency may remain silent; hence, it is to be emphasized that the screening should be universal given the high incidence in this study population if subsequent morbidity related to hemolysis following oxidative stressors are to be avoided. Further, Hambantota and Matara districts in the Southern province were previously considered as malaria endemic areas and it is noteworthy that higher percentages of babies were detected from this area.

This disorder also fits into the criteria for screening laid down by Wilson and Jungner in 1962 for the implementation of newborn screening (21). It is sufficiently common, has a robust diagnostic test, 
if undetected can lead to adverse consequences of hemolysis and renal shut down, and can be managed with ease. The cost-effectiveness for the diagnosis of this disease is such that it does not require active management in a resource-poor setting. Since the blood of dried blood spot on a filter paper is enough, transport is easy to a regional center, rather than multiple laboratories, across the country that may serve the purpose. We emphasize the need for expanding the panel of NBS to include G6PD deficiency as an important public health perspective. The high incidence observed and possible disastrous sequalae may make it an important candidate disorder for NBS panel with an added advantage as it requires no long-term cost for management.

\section{Conclusions}

In conclusion, we conducted a comprehensive newborn screening of G6PDD in a large cohort of population from Southern Sri Lanka. The cut-offs we have defined at 6 months may be at a disadvantage with high attrition but it saves the cost of additional genetic analysis.

\section{Acknowledgements}

We acknowledge the assistance of all medical and nursing officers attached to the hospitals for their contribution by giving continuous support in sample collection and follow up of babies. Further, members of the Nuclear Medicine Unit who conducted sample analysis and subject follow up are greatly appreciated.

\section{Funding}

All expenditure (reagents for the study and temporary staff payments) was from the Development fund of the Nuclear Medicine Unit, University of Ruhuna

\section{References}

1. De Jesus VR, Mei JV, Cordovado SK, et al. The Newborn Screening Quality Assurance Program at the Centers for Disease Control and Prevention: Thirty-five Year Experience Assuring Newborn Screening Laboratory Quality. International Journal of Neonatal Screening, 2015; 1(1): 13-26.
2. De Jesus VR, Mei JV, Bell CJ. Improving and assuring newborn screening laboratory quality worldwide: 30 -year experience at the Centers for Disease Control and Prevention. Semin. Perinatol, 2010; 34: 125-33.

3. Mason PJ, Bautista JM, Gilsanz F. G6PD deficiency: the genotype-phenotype association. Blood Review, 2007; 21: 267-83.

4. Goyal M, Garg A, Goyal MB, Kumar S. et al. Newborn screening for G6PD deficiency: A 2-year data from North India. Indian J Public Health, 2015; 59: 145-8.

5. Mezzacappa MA, Facchini FP, Pinto AC, Cassone AE, Souza DS, Bezerra MA, et al. Clinical and genetic risk factors for moderate hyperbilirubinemia in Brazilian newborn infants. J Perinatol, 2010; 30: 819-26.

6. Frank JE. Diagnosis and Management of G6PD Deficiency. Am Fam Physician , 2005; 72: 1277-82.

7. Azma RZ, Hidayati N, Farisah NR, et al. G6PD enzyme activity in normal term Malaysian neonates and adults using a OSMMR2000-D kit with $\mathrm{Hb}$ normalization. Southeast Asian J Trop Med Public Health, 2010; 41(4): 982-88.

8. Mohanty D, Mukherjee MB, Colah RB. Glucose-6phosphate dehydrogenase deficiency in India. Indian $J$ Pediatr, 2004; 71:525-9.

9. Amarasena S, Hettiarachchi M. Establishment of National programme on newborn screening for congenital hypothyroidism. Sri Lanka Journal of Diabetes Endocrinology and Metabolism, 2018; 8(2), 13-8.

10. Anderle A, Bancone G, Domingo GJ. et al. Point-of-Care Testing for G6PD Deficiency: Opportunities for Screening. Int. J. Neonatal Screen 2018; 4: 34.

11. Beutler E. The genetics of glucose-6-phosphate dehydrogenase deficiency. Semin. Haematol, 1990; 27: 137-64.

12. Alkhotani A, Eldin MN, Zaghloul A. Evaluation of neonatal jaundice in the Makkah region. Sci Rep, 2014; 4: 4802 .

13. Kaplan M, Hammerman C, Vreman HJ. Acute haemolysis hyperbilirubinaemia in glucose-6-phosphate dehydrogenase deficient heterozygotes. J Paediatr, 2001; 139: $137-40$.

14. Gregg XT, Prchal JT. Red cell enzymopathies. In: Hoffman R, ed. Hematology: basic principles and practice. $4^{\text {th }}$ ed. Philadelphia: Churchill Living stone, 2000: 657-60. 
15. Reclos GJ, Schulpisb KH, Gavrilic S. Evaluation of glucose-6-phosphate dehydrogenase activity in two different ethnic groups using a kit employing the haemoglobin normalization procedure. Clinical Biochemistry, 2003; 36:393-5.

16. World Health Organization (2016). Technical Specifications Series for Submission to WHO Prequalification-Diagnostic Assessment: In Vitro Diagnostics Medical Devices to Identify Glucose-6Phosphate Dehydrogenase (G6PD) Activity. WHO, Geneva.

17. Watchko JF. Screening for glucose-6-phosphate dehydrogenase deficiency in newborns-practical considerations. J. Pediatr, 2002; 161, 179-80.
18. Premawardhena A, Perera PS. Anaemia in Sri Lanka: the missing pieces. Ceylon Medical Journal, 2018; 63: 105-7.

19. Bisoi S, Chakraborty S, Chattopadhyay D. Glucose-6phosphate dehydrogenase screening of babies born in a tertiary care hospital in West Bengal. Indian J Public Health, 2012; 56: 146-8.

20. Joshi SR, Patel RZ, Patel HR. High prevalence of G6PD deficiency in Vataliya Prajapati Community in western India. Haematologia (Budap), 2001; 31: 57-60.

21. Dobrow MJ, Hagens V, Chafe R. Consolidated principles for screening based on a systematic review and consensus process. CMAJ: Canadian Medical Association Journal, 2018; 190(14): E422-E429. 